\title{
SELF ASSESSMENT QUESTIONS
}

\section{A case of migratory lymphadenopathy and cutaneous anergy in an Asian woman}

\author{
Haider M Al Attia, Yasser H Al Ahmed, Afaf El Hag, Rima N El Abassi
}

Answers on $p 663$.

Department of
Internal Medicine and
Histopathology,
Mafraq Hospital, Abu
Dhabi, United Arab
Emirates
H M Al Attia
Y H Al Ahmed
A El Hag
R N El Abassi

Correspondence to: Dr Haider M Al Attia, Mafraq Hospital, PO Box 2951, Abu Dhabi, UAE

Submitted 9 March 1999 Accepted 14 October 1999

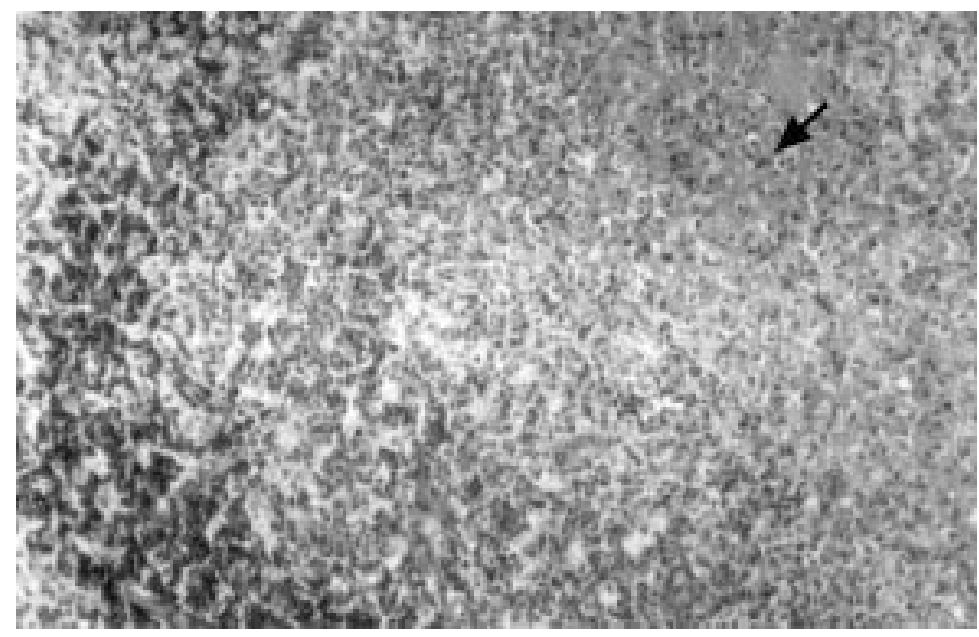

Figure 1 Area of karyorrhectic necrosis (arrow) surrounded by histiocytes (pale zone), lymphoctyes, and plasma cells (dark zone).

A 40 year old Pakistani woman presented with one month history of fever, night sweats, and progressive and painful swelling in the right side of the neck. She was febrile (temperature $38-40^{\circ} \mathrm{C}$ ). Right deep cervical, occipital, and jugulodiagastric lymph nodes were palpable, firm, and tender. Save for a moderately severe asymmetrical arthralgia of right wrist, shoulder and proximal interphalangeal joints, the rest of her physical examination was unremarkable. Laboratory data showed, mild leucopenia of $3.6 \times 10^{9} / 1$ with $64 \%$ neutrophils, $26 \%$ lymphocytes (no atypical form), and 10\% monocytes, erythrocyte sedimentation rate (ESR) 64 $\mathrm{mm} /$ hour, and $\mathrm{C}$ reactive protein of $105 \mathrm{mg} / 1$ (normal $<10 \mathrm{mg} / \mathrm{l}$ ). A skin test for tuberculin reactivity was negative. Results of chest radiography, blood chemistry, urine and stool analysis, throat swab, blood cultures, Venereal Disease Research Laboratory test, test for HIV, heterophil antibodies, and serological studies for salmonella serotypes, brucellosis, toxoplasmosis, Lyme disease, Epstein-Barr virus, cytomegalovirus, human $\mathrm{T}$ cell leukaemia enia of $3.1 \times 10^{9} / 1$ with $7 \%$ monocytes and ESR of $36 \mathrm{~mm} /$ hour were documented. All the tests that had been carried out during her previous admission were repeated. The results showed no abnormal findings. The tuberculin test was again negative. Biopsy of a left deep cervical node showed identical changes to those of the previous biopsy. She was conservatively managed with bed rest and administration of paracetamol. The fever subsided completely after 10 days and lymphadenopathy resolved in two weeks. The patient remains well to date. During the follow up, the tuberculin test was repeatedly found to be positive.

\section{Questions}

(1) What was the overall diagnosis?

(2) What two unusual features are described in this case? 


\section{Multiple focal lesions in liver and spleen in acute leukaemia}

Sangeet Ghai, Sanjay Thulkar, Prakash Neduvelil Purushothaman, Sanjay Sharma

Answers on $p 664$.

An 18 year old girl presented with a two month history of fever and bleeding tendency. On examination, she had multiple petechial haemorrhages all over her body. There

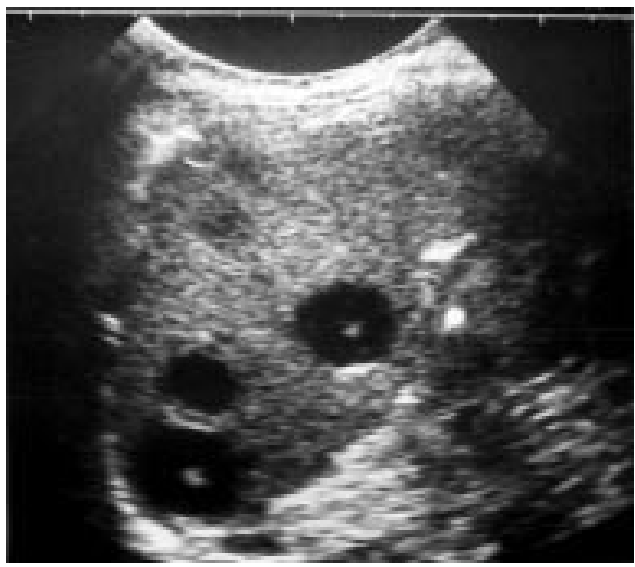

Figure 1 Sonogram of the spleen shows multiple, rounded hypoechoic lesions, some with typical bull's eye appearance.

Institute Rotary Cancer Hospital, All India Institute of Medical Sciences, New Delhi 110 029, India: Department of Radio-diagnosis S Ghai

$S$ Thulkar

S Sharma

\section{Department of} Medical Oncology P N Purushothaman

Correspondence and reprint requests to: Dr Sanjay Sharma, A-86, Inderpuri, New Delhi 110012, India (email:

ssh@medinst.ernet.in)

Submitted 1 June 1999 Accepted 8 October 1999

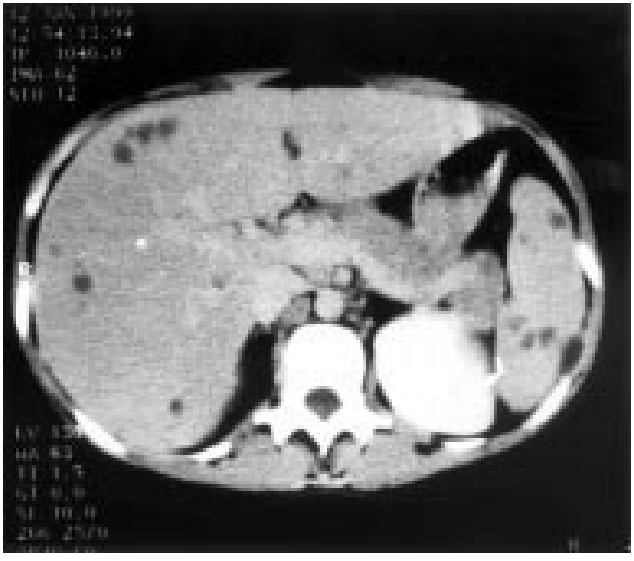

Figure 2 Computed tomogram of the upper abdomen showing multiple small low attenuation lesions in the liver and spleen. Incidentally old calcified granulomas are also seen in liver and spleen.

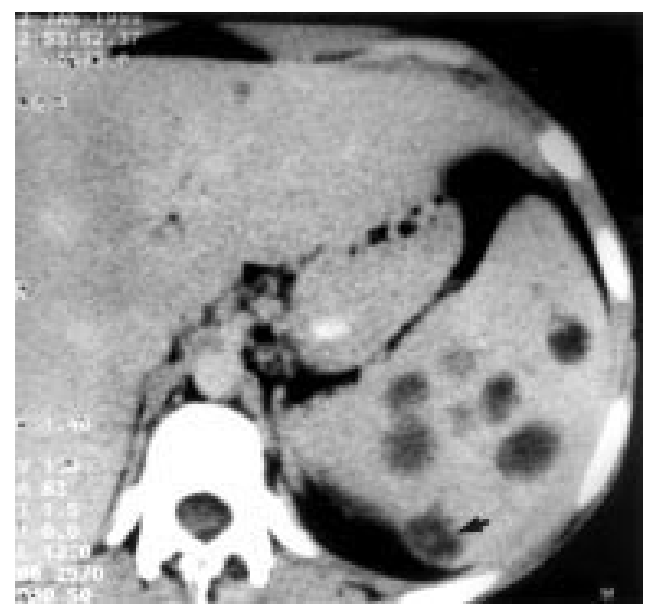

Figure 3 Computed tomogram shows multiple, low attenuation, rounded lesions in the spleen. One of the lesions (arrow) also shows a central nidus of high attenuation.

was no organomegaly or peripheral lymphadenopathy. Systemic examination was unremarkable. Total leucocyte count was $117 \times 10^{9} / 1$ and platelet count $45 \times 10^{9} / 1$. Peripheral smear showed presence of blast cells. Bone marrow biopsy revealed $60 \%-70 \%$ myeloblasts. The patient was diagnosed as having myeloid leukaemia. After two cycles of chemotherapy, the patient went into remission.

After three weeks, she became febrile again and developed diffuse upper abdominal tenderness and jaundice. Abdominal sonography (fig 1) and computed tomography (figs 2 and 3) were performed. Total leucocyte count at this time was $9.5 \times 10^{9} / 1$.

\section{Questions}

(1) What are the findings on ultrasound and computed tomography?

(2) What is the radiological diagnosis?

(3) How can the diagnosis be confirmed? 


\title{
A pregnant patient with bilateral ischaemic limbs
}

\author{
N B Teo, N Mamode, D P Leiberman
}

Answers on p 666.

A 29 year old pregnant woman, at 35 weeks' gestation, was admitted with a three day history of a painful left thigh and calf which was exacerbated by walking. She also complained of paraesthesiae, muscle weakness, and coldness of the left foot which at times became cyanosed. These symptoms were associated with dyspnoea on exertion.

When admitted to the obstetric ward, the left lower limb pulses were impalpable. The left lower leg was mottled and cold but was viable.

Peripheral Vascular Unit, Royal Infirmary Glasgow, Glasgow, UK $\mathrm{N}$ B Teo

N Mamode

D P Leiberman

Correspondence to: Mr N B Teo, Pathology Department, University of Liverpool, Duncan Building, Daulby Street, Liverpool L69 3GA, UK

(email:

teonb@liverpool.ac.uk)

Submitted 27 August 1999 Accepted 8 October 1999

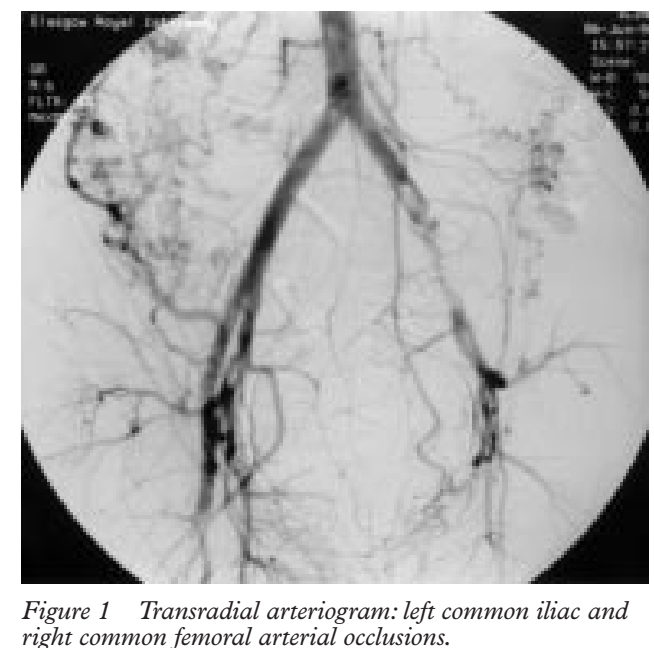

right common femoral arterial occlusions.
The electrocardiogram showed ST changes in the septal leads with $\mathrm{R}$ waves in V1 suggestive of right ventricular hypertrophy as well as $\mathrm{T}$ wave inversion in the anterolateral leads thought by the cardiologist to be suggestive of pulmonary thromboembolism. Chest radiography showed cardiomegaly. Full blood count, concentrations of urea and electrolytes, and thrombophilia screen were normal.

On review by the vascular team, it was noted that apart from a very weak right femoral pulse, there was absence of pulses in both lower limbs, which were significantly ischaemic.

After cardiological review, a transthoracic echocardiogram showed a large right ventricle with a dilated pulmonary artery. No atrial septal defect was seen and there was no evidence of thrombus.

Doppler ultrasound showed a $10 \mathrm{~cm}$ left popliteal venous thrombus as well as occlusion of bilateral common femoral and superficial arteries. This was followed by a transradial arteriogram that showed the thrombus causing a left common iliac occlusion and a right common femoral arterial occlusion (fig 1).

\section{Questions}

(1) What is the diagnosis?

(2) Describe the pathogenesis of this clinical condition?

(3) How should the diagnosis be made?

(4) Describe the most recent development in the diagnosis of this condition?

(5) What is the treatment?

\section{Severe symptomatic hypercalcaemia}

\author{
José María Calvo-Romero, María del Carmen Bonilla-Gracia
}

Answers on $p 668$.

\section{Internal Medicine Service, Infanta Cristina University Hospital, Badajoz, Spain \\ J M Calvo-Romero M C Bonilla-Gracia}

Correspondence to: José María Calvo-Romero, Héroes de Cascorro 9, 31A, 06004 Badajoz, Spain

Submitted 7 July 1999 Accepted 8 October 1999
A 49 year old man had a six week history of depression, constipation, proximal muscle weakness, anorexia, and weight loss of about 20 $\mathrm{kg}$. The patient was not taking any medication and there was no history of nephrolithiasis, peptic ulcer, headache, or visual defects. Physical examination showed light proximal muscle weakness without atrophy, dehydration, and no other remarkable findings. The blood pressure was $140 / 85 \mathrm{~mm} \mathrm{Hg}$. The patient's blood chemical values were: glucose $5.2 \mathrm{mmol} / \mathrm{l}$, urea nitrogen $13.2 \mathrm{mmol} / \mathrm{l}$, creatinine $141 \mu \mathrm{mol} / \mathrm{l}$, sodium $136 \mathrm{mmol} / 1$, potassium $4.1 \mathrm{mmol} / 1$, chloride $101 \mathrm{mmol} / \mathrm{l}$, phosphorus $1.3 \mathrm{mmol} / \mathrm{l}$, magnesium $0.98 \mathrm{mmol} / \mathrm{l}$, alkaline phosphatase $115 \mathrm{U} / 1$, creatine kinase $82 \mathrm{U} / 1$, protein $76 \mathrm{~g} / \mathrm{l}$, and albumin $43 \mathrm{~g} / 1$. The blood calcium was 4.8 $\mathrm{mmol} / \mathrm{l}$, confirmed by repeated determinations. The 24 hour urinary calcium excretion was 12.3 mmol. An electrocardiogram revealed sinus rhythm and shortened QT interval. Free thyroxine and thyrotrophin serum concentrations were normal. Serum intact parathyroid hormone was $488 \mu \mathrm{g} / 1$ (normal values $10-65 \mu \mathrm{g} / \mathrm{l}$ ).

\section{Questions}

(1) What is your differential diagnosis for this case?

(2) What further investigations would you perform?

(3) What is the therapy for the severe hypercalcaemia in this case? 


\section{SELF ASSESSMENT ANSWERS}

A case of migratory lymphadenopathy and cutaneous anergy in an Asian woman

Q1: What was the overall diagnosis?

Histiocytic necrotising lymphadenitis (Kikuchi Fujimoto disease)

\section{Q2: What two unusual features are described in this case?}

(1) The transient cutaneous anergy during the acute phase of the illness and (2) the migratory pattern of lymphadenopathy after a prolonged remission.

\section{Discussion}

Histiocytic necrotising lymphadenitis or Kikuchi Fujimoto disease (KFD) is an uncommon, self limiting disease that primarily affects the cervical lymph nodes in young adults, mainly females. The aetiology seems unknown but an infective origin seems likely. Though the condition commonly affects patients of Asian descent, it has been more and more seen in other populations. ${ }^{12}$

KFD is a peculiar condition with broad morphological spectrum that can readily be mistaken for malignant lymphoma or lupus, ${ }^{3}$ thus an accurate distinction is crucial. Patients usually develop painless, often unilateral cervical lymphadenopathy in the context of fever, night sweats, arthralgia, rash, and asthenia. ${ }^{4}$ Painful lymphadenopathy may also develop. ${ }^{3}$ Generalised lymphadenopathy occurs infrequently ${ }^{3}$ and has recently been associated with human $\mathrm{T}$ cell leukaemia virus-1 infection. ${ }^{5}$ The true nature of the lesion becomes evident on a lymph node biopsy rather than fine needle aspiration samples. ${ }^{6}$ Other infectious agents of possible relevance to pathogenesis include herpes virus 6, EpsteinBarr virus, cytomegalovirus, parvovirus B-19, Yersinia enterocolitica, and brucella species. ${ }^{7-12}$ $\mathrm{KFD}$ has been repeatedly linked with systemic lupus erythematosus ${ }^{3-13}$ and described in association with Hashimoto's thyroiditis, ${ }^{13}$ Sweet's syndrome, ${ }^{14}$ and Still's disease. ${ }^{15}$ The condition has a low recurrence rate of $3 \%-4 \%$ and rarely recurs after a prolonged remission. The acute phase of illness usually responds to bed rest and symptomatic treatment. The symptoms resolve within 1-4 months; however there have been cases that recurred $^{3}$ or persisted as long as a year. ${ }^{4}$

In this case the migratory pattern of cervical lymphadenopathy, which was painful and tender at first presentation and painless on relapse, has led to a clinical diagnostic dilemma; a situation that would have not been settled without a second lymph node biopsy. Such a migratory characteristic, however, has not been specifically referred to in the previous literature. KFD should be considered in the differential diagnosis of any clinical setting with migratory lymphadenopathy. The repeatedly negative tuberculin test during acute illness was another issue of interest. Unless the cellular mediated immune reaction is stunted, the tuberculin test is expected to be positive in an Asian adult, even in the absence of active tuberculous disease. Thus, the negative reaction in our patient was likely to be a sign of transient cutaneous anergy possibly induced by the disease activity and recovered during remission. To the best of our knowledge this phenomenon was also not reported before, and is worthy of further study in future cases.

\section{Final diagnosis}

Histiocytic necrotising lymphadenitis (Kikuchi Fujimoto disease).

1 Simonsen K, StormT L. Histiocytic necrotizing lymphadenitis (Kikuchi's disease). Ugeskr Laeger 1991;153:2836-7.

2 Depree C, Pelte MF, Delacretaz F, et al. Histiocytic necrotizing lymphadenitis or Kikuchi's disease. Anatomoclinical study of 4 cases. Nouv Rev Fr Hematol 1990;32:2417 .

3 Kuo TT. Kikuchi's disease (histiocytic necrotizing lymphadenitis). A clinicopathologic study of 79 cases with an analysis of histologic subtypes, immunohistology, and DNA sis of histologic subtypes, immunohistol.
ploidy. Am $\mathcal{J}$ Surg Pathol 1995;19:798-809.

4 Dylewski J, Berry G, Pham-Dang H. An unusual cause of cervical lymphadenitis: Kikuchi-Fujimoto disease. Rev Inf Dis 1991;13:823-5.

5 Bataille V, Harland CC, Behrens J, et al. Kikuchi disease (histiocytic necrotizing lymphadenitis) in association with HTLV 1. Br F Dermatol 1997;136:610-12.

6 Kung IT, Ng WF, Yuen RW, et al. Kikuchi's histiocytic lymphadenitis. Diagnosis by fine needle aspiration. Acta Cytolocica 1990;34:323-6.

7 Hoffman A, Kirn E, Kuerten A, et al. Active human herpesvirus-6 (HHV-6) infection associated with KikuchiFujimoto disease and systemic lupus erythematosus. In vivo 1991;5:265-9.

8 Yen A, Fearneyhough P, Raimer SS, et al. EBV- associated Kikuchi's histiocytic lymphadenitis with cutaneous manifestations. F Am Acad Dermatol 1997;36:342-6.

9 Biasi D, Caramaschi P, Carletto A, et al. Three clinical Biasi D, Caramaschi P, Carletto A, et al. Three clinical
reports of Kikuchi's lymphadenitis combined with systemic reports of Kikuchi's lymphadenitis combined with syst
lupus erythematosus. Clin Rheumatol 1996;15:81-3.

10 Yufu Y, Matsumoto M, Miyamura T, et al. Pavovirus B19-associated haemophagocytic syndrome with lymphadenopathy resembling histiocytic necrotizing lymphadenitis (Kikuchi's disease). Br F Haematol 1997;96:868-71.

11 Heikens J, Tel W, van de Stadt J, et al. Kikuchi's lymphadenitis: report of a Yersinia enterocolitica-associated case and an overview of aetiology and clinical outcome. Neth f Med 1992;41:222-8.

12 Rodr'iguez Martorell J, Mart'in MV, B'aez JM, et al. Kikuchi-Fujimoto necrotizing lynphadenitis associated with brucellosis. Sangre 1992;37:201-4.

13 Bousquet E, Tubery M, Brousset P, et al. Kikuchi syndrome, Hashimoto thyroiditis and lupus serology. A propos of a case. Rev Med Interne 1996;17:836-8.

14 Itoh H, Shimasaki S, Nakashima A, et al. Sweet's syndrome Itoh $\mathrm{H}$, Shimasaki S, Nakashima A, et al. Sweet's syndrome
associated with subacute necrotizing lymphadenitis. Intern associated with subacu

15 Ohta A, Matsumoto Y, Ohta T, et al. Still's disease associated with necrotizing lymphadenitis (Kikuchi's disease): report of 3 cases. F Rheumatol 1988;15:981-3. 
Multiple focal lesions in liver and spleen in acute leukaemia

Q1: What are the findings on ultrasound and computed tomography?

Abdominal ultrasound shows multiple hypoechoic focal lesions in the spleen, some of them having central echogenic foci producing bull's eye or target configuration. Contrast enhanced computed tomography shows multiple, nonenhancing, hypodense focal areas in liver in addition to the spleen. Few of the splenic lesions demonstrate central hyperdense foci. Incidentally, a few old healed calcified foci are also seen in the liver and spleen.

Q2: What is the radiological diagnosis?

The image morphology of liver and splenic lesions in this immunocompromised patient is strongly suggestive of fungal infection.

Q3: How can the diagnosis be confirmed? Image guided fine needle aspiration and microscopic examination of the aspirate can be done to confirm the diagnosis. An aspirate from the centre of the focal lesion is most likely to yield a positive result as the fungal elements are most abundant in these central necrotic areas. Blood and tissue cultures may be falsely negative, particularly with candidal infections. In our patient, ultrasound guided fine needle aspiration was performed. The aspirate demonstrated mycelia and budding yeast cells confirming the diagnosis of hepatosplenic candidiasis.

\section{Discussion}

Fungal infections of the liver and spleen occur almost exclusively in individuals with underlying defects of host immune defence mechanism. The most commonly implicated organism is Candida albicans, but infections with other fungi such as aspergillus and cryptococcus may also occur. ${ }^{1}$ The presenting symptoms are generally non-specific, consisting of fever, pain referable to the area of involvement, tenderness on direct palpation, enlargement of liver and/or spleen, and rarely, jaundice. Usually both liver and spleen are involved, though either organ may be affected in isolation. $^{2}$

Initially hepatomegaly and/or splenomegaly are present. Subsequently focal lesions develop that later spread throughout the parenchyma. They may be single or multiple. When multiple, they tend to be located adjacent to one another or may become partially confluent. ${ }^{3}$ Their size varies from $0.3 \mathrm{~cm}$ to 4.0 $\mathrm{cm}$ and they have relatively well defined borders. Five sonographic patterns of hepatosplenic candidiasis have been described. ${ }^{3}$ Pattern 1 represents an early active phase of the disease in which ultrasound demonstrates either a "wheel within wheel" (type a) or "wagon wheel" (type b) appearance. In the former appearance, the outer hypoechoic rim is formed by fibrosis while the inner hyperechoic rim is composed of the inflammatory process. In the centre of the inner hyperechoic zone, there is an area of necrosis identified as the
Box 1: Various patterns of candidal infection of liver and spleen on ultrasound

- Pattern 1a: wheel within wheel appearance

- Pattern 1b: wagon wheel appearance

- Pattern 2: bull's eye appearance/target configuration

- Pattern 3: pure hypoechoic defect

- Pattern 4: echogenic lesion with a varying intensity of posterior acoustic shadow

Box 2: Differential diagnosis of multiple hypodense/hypoechoic splenic and liver lesions

- Lymphoma

- Leukaemia

- Metastatic disease

- Bacterial infection

- Fungal infection

- Parasitic infection

- Sarcoid

hypoechoic nidus. In the wagon wheel appearance, echogenic radial strands are seen which imitate the spokes of a wheel. These "spokes" represent the inflammatory process, whereas hypoechoic regions between the spokes is the fibrous component. The axis of the wheel is formed by hypoechoic, necrotic nidus.

Pattern 2, manifested by the "bull's eye" or target configuration, lacks the central necrotic hypoechoic nidus. The lesion consists of the inflammatory process forming the echogenic centre, which is surrounded by fibrosis seen as the hypoechoic rim. ${ }^{3}$ Pattern 3 is characterised by a purely hypoechoic lesion. It is seen when the inflammatory process is being replaced by fibrosis. ${ }^{3}$ Later this hypoehoic lesion is transformed into a completely echogenic lesion (pattern 4) with a varying degree of posterior acoustic shadow. This sonographic appearance is produced by scar tissue with or without calcification. This echogenic lesion is usually smaller than pattern 2 or 3 lesions and may even disappear completely in the course of the healing process. ${ }^{3}$

In acute phase of the disease, lesions of patterns 1 and 2 prevail. As disease progresses, pattern 3 lesions are identified, however, pattern 1 and 2 lesions may still be present. When pattern 4 lesions appear, pattern 1 is no longer seen and the lesions of other patterns regress in size. This is recognised late in the course of the disease. ${ }^{3}$

Fungal abscesses in liver and spleen of neutropenic patients are not always detectable on sonography, even in the presence of disseminated infection. The lesions become apparent when the neutrophil count returns to normal. ${ }^{4}$ It is important to identify pattern 1 or 2 in at least one lesion, as these appear only when the 
neutrophil count in a previously neutropenic patient is returning to normal and infection is active. ${ }^{5}$ This fact suggests that the inflammatory response of the host plays a part in defining the characteristic appearance. ${ }^{6}$ Pattern 3 and 4 lesions occur later in course of the disease and suggest that the infection is subsiding. ${ }^{5}$

Computed tomography may demonstrate similar appearances. Pastakia et al reported that pattern 1 lesions were not seen on computed tomograms and pattern 2 lesions were demonstrated only occasionally. ${ }^{5}$ Pattern 3 lesions (multiple rounded areas of decreased attenuation scattered throughout the liver and spleen) were most common. Pattern 4 lesions, representing areas of calcification, were seen late in course of the disease. In our patient, no focal hepatic lesion could be detected on ultrasound, while computed tomography showed multiple pattern 3 and one pattern 2 lesion in the liver and multiple pattern 2 and 3 lesions in the spleen. Similar observation was also made by Pastakia et al. A computed tomogram is more sensitive in detecting the focal lesions; however, it is less specific as the characteristic pattern 1 and 2 lesions are demonstrated only occasionally. Periportal areas of increased attenuation seen on computed tomography are also reported which correlate pathologically with focal linear fibrosis in these immunocompromised patients. ${ }^{5}$

The usual differential diagnosis of multiple, focal lesions in liver and spleen include lymphoma, leukaemia deposits, metastasis, bacterial and fungal infection, and sarcoid. Most of these diseases give rise to non-specific focal hypoechoic lesions on sonography. Target lesions may however be seen in metastatic disease, although metastatic disease is unusual in the spleen. Deposits of lymphoma and leukaemia show rapid regression after cytostatic treatment, a fact which may help to differentiate them from the lesions of other aetiologies. ${ }^{6}$

To summarise, the detection of focal hepatic and splenic lesions with characteristic image morphology should suggest a possible underlying fungal disease in a febrile leukaemic patient. Apart from the aetiological diagnosis of fungal infection, the imaging features also provide understanding of their evolution over time.

\section{Final diagnosis}

Multiple candidal abscesses in liver and spleen in acute myeloid leukaemia.

\footnotetext{
1 Berlow ME, Spirit BA, Weil L. CT follow-up of hepatic and splenic fungal micro-abcesses. F Comput Assist Tomogr splenic fungal

2 Maxwell AJ, Mamtora H. Fungal liver abcesses in acute leukemia_a report of two cases. Clin Radiol 1988;39:197201 .

3 Grunebaum M, Ziv N, Kaplinsky C, et al. Liver candidiasis - the various sonographic patterns in the immunocompromised child. Pediatr Radiol 1991;21:497-500.

4 Chew FS, Smith PL, Barboriak D. Candidal splenic abcesses. AfR Am f Roentgenology 1991;156:474.

5 Pastakia B, Shawker TH, Thaler M, et al. Hepatosplenic candidiasis: wheels within wheels. Radiology 1988;166:41721.

6 Gorg C, Wiede R, Schwerk WB, et al. Ultrasound evaluation of hepatic and splenic microabscesses in the immunocompromised patient: sonographic patterns, differential diagnosis and follow-up. F Clin Ultrasound 1994;22:525-9.
} 


\section{A pregnant patient with bilateral} ischaemic limbs

Q1: What is the diagnosis?

The patient has paradoxical embolism and patent foramen ovale.

\section{Q2: Describe the pathogenesis of this clinical condition?}

A favourable right to left pressure gradient, secondary to raised right atrial pressure (RAP), must exist to promote shunting of venous thrombi through the intracardiac defect. Pulmonary thromboembolism is the most common cause of acute elevation of RAP. Occlusion of left pulmonary artery causes a rise in mean pulmonary arterial pressures with a simultaneous fall in systemic arterial pressure. Favourable pressure gradient only exists when at least one third of the pulmonary arterial tree is occluded or when the mean pulmonary arterial for right to left shunting at least $30 \mathrm{~mm}$ Hg. ${ }^{1}$

\section{Q3: How should the diagnosis be made?}

Four criteria have to be met for diagnosis: (1) deep vein thrombosis and/or pulmonary thromboembolism, (2) an abnormal communication between the venous systemic circulation, (3) clinical, angiographic, and pathological evidence for systemic embolism, and (4) pressure gradient allowing right to left shunting at some point in the cardiac cycle. ${ }^{1}$ Clinically, the diagnosis of paradoxical embolism is presumptive, relying on circumstantial evidence as well as a high index of suspicion. Premorbid diagnosis of patent foramen ovale is usually made by transthoracic or transoesophageal colour Doppler echocardiography (TTE or TOE). Studies as well as our case confirm the superiority of TOE over TTE in detecting patent foramen ovale. ${ }^{2}$ For the diagnosis, Chen et al reported that TOE had a sensitivity of $100 \%$ and a specificity of $97 \%$, while they were $63 \%$ and $78 \%$ respectively for $\mathrm{TTE}^{2}$

In this case, postoperative TOE revealed a large atrial septal defect with a free left to right flow and thrombus in the right middle pulmonary artery.

\section{Q4: Describe the most recent development in the diagnosis of this condition?}

It was suggested that contrast echocardiography is a useful and probably more effective manoeuvre to exclude patent foramen ovale. ${ }^{1}$ It involves high pressure injection into the venous circulation of a saline solution containing microbubbles in suspension. A valsalva manoeuvre can increase the sensitivity of the contrast study. However, it was also reported that the cough test is superior to the valsalva manoeuvre in the contrast study.

Recently, contrast transcranial Doppler (TCD) was shown to have a sensitivity and specificity of $100 \%$ in comparison with contrast TOE. ${ }^{1}$ Therefore, contrast TCD can be an alternative method for detection of right to left shunting.

\section{Q5: What is the treatment?}

Our patient was started on intravenous heparin infusion. She was taken to theatre for an emergency caesarean section and bilateral femoral embolectomies. The baby was delivered uneventfully. Anticoagulation was continued postoperatively and the mother made an uncomplicated recovery. It is planned to close the atrial septal defect in the near future.

As part of acute management, ${ }^{3}$ most authors agree that immediate anticoagulation should be started in the absence of any contradiction. Thrombolysis or embolectomy is indicated to treat peripheral embolism which threatens limb viability. In suspected cases of paradoxical embolism, thrombolysis is indicated in the presence of both pulmonary thromboembolism and acute cor pulmonale.

Thrombolysis can reduce RAP and minimise recurrence of paradoxical embolism. ${ }^{1}$ As for the patient who is haemodynamically compromised, pulmonary embolectomy should be performed if indicated and feasible. Rarely, impending paradoxical embolism is best managed with intracardiac embolectomy and closure of patent foramen ovale. ${ }^{1}$

Current long term therapeutic options are: (1) long term anticoagulation therapy, (2) long term antithrombotic therapy, (3) surgical closure of patent foramen ovale either open heart surgery or transcatheter placement of the double umbrella device, or (4) inferior vena cava (Greenfield) filter. Until now, there has been little information regarding the long term outcome of any particular treatment modality. ${ }^{1}$

\section{Discussion}

For venous thromboses to travel into the systemic circulation the clot has to bypass the pulmonary bed by passage through an abnormal communication. This may be a fixed atrial septal defect or a patent foramen ovale which allows right to left shunting when the right atrial pressure RAP is raised by a pulmonary thromboembolism. ${ }^{1}$ It was first diagnosed in 1877 by Cohnheim, ${ }^{4}$ and although cases are not infrequently reported, it was rarely described in pregnancy. ${ }^{5}$

Aburahma reported that $56 \%$ of cases of emboli had probable or possible paradoxical embolism, ${ }^{3}$ while Caplan et al reported that $36 \%$ of embolic strokes had an unidentifiable cardiac source. ${ }^{6}$ Importantly, Lechat et al showed that patent foramen ovale was found in $40 \%$ of patients with unexplained embolic stroke compared with $10 \%$ in a control group. ${ }^{7}$ Patent foramen ovale occurs in $11 \%$ to $35 \%$ of the normal population and grows larger with age. ${ }^{8}$

In our case, the clinical features were strongly suggestive of a large pulmonary thromboembolism in association with acute peripheral arterial ischaemia, giving a strong suspicion of the diagnosis of paradoxical embolism. It is interesting that the TTE did not suggest paradoxical embolism but the postoperative TOE did show the atrial septal defect and clot in the pulmonary artery. This is in keeping with studies which have shown the 


\section{Learning points}

- Prevalence of paradoxical embolism as the cause of peripheral or cerebral embolic events has been under-estimated

- There is right to left shunting through atrial septal defect or patent ductus arteriosus when right atrial pressure is elevated by pulmonary thromboembolism

- Transoesophageal echocardiogram is superior to transthoracic echocardiogram in detecting patent foramen ovale

- Angiography is safe in the third trimester and magnetic resonance arteriography is less invasive

- Thrombolysis reduces right atrial pressure and prevents recurrence of patent ductus arteriosus

- In pregnancy, surgical embolectomy is a safer option

- Acute management requires a high index of suspicion, early diagnosis, and timely intervention with a multidisciplinary approach

superiority of TOE against TTE in detecting patent foramen ovale. ${ }^{2}$

The contrast echocardiogram, especially with the valsalva manoeuvre and cough test, is useful for excluding patent foramen ovale. ${ }^{1}$ Contrast transcranial Doppler can be an alternative method for detection of right to left shunting. We showed the extent of the peripheral clot in our patient by angiography, which is safe for the fetus in the third trimester, but we had considered magnetic resonance angiography, which is less invasive and also safe in pregnancy. ${ }^{9}$

Normally, management of patients with paradoxical embolism would be determined by the need to re-establish peripheral flow, if possible; to prevent further arterial embolisation; and to reduce the haemodynamic threat from the pulmonary embolus. This may require that, in addition to immediate anticoagulation, both venous and peripheral thrombolysis should be considered. We felt that thrombolysis, which has been advocated to reduce RAP and minimise recurrence of paradoxical embolism, would threaten the pregnancy, ${ }^{10}$ and that surgical embolectomy and caesarean delivery would offer the safest solution. Placement of an inferior vena cava filter before section to prevent further pulmonary emboli was considered but as the pelvic veins were patent on ultrasound, it was considered unnecessary. After successful delivery, the TOE confirmed atrial septal defect and the patient is awaiting surgical closure at present. The complexity of this case-which requires specialist obstetric, imaging, surgical, intensive care, and anaesthetic expertise-could be used as an argument for locating obstetric units within general hospital centres.

It is interesting to speculate about the outcome if the peripheral embolus had lodged in the right internal iliac as well as the left. The uterine artery supplying the placenta would have been occluded with a probable intrauterine death. Fortunately, however, this was not the case and both the mother and baby have done well.

The high prevalence of clinically occult deep vein thrombosis and the presence of patent foramen ovale in up to $35 \%$ of the population highlights the fact that paradoxical embolism may be the cause of a peripheral or cerebral embolic event more often than is currently suggested.

The key points of management are the need for high index of suspicion, early diagnosis, and timely intervention with a multidisciplinary approach. The long term treatment of paradoxical embolism is less well defined. More studies are needed to assess the risk of recurrent arterial ischaemic events in the presence of patent foramen ovale as well as to examine the long term outcome of the respective treatment strategies.

\section{Final diagnosis}

Paradoxical embolism and patent foramen ovale.

1 Robert RM III, A Stacey H, Michael SB, et al. Impending PDE Arch Intern Med 1998;158:438-48.

2 Chen W, Kuan P, Lien W, et al. Detection of patent foremen ovale by contrast transechocardiogram. Chest 1992;101: ovale by

3 Aburahma AF, Lucentre FC, Boland JP. Paradoxical embolism: an under-estimated entity - a plea for comprehensive workup. F Cardiovasc Surg (Torino) 1990;31:685-92.

4 Cohnheim J. Thrombose und Embolid. Vorlesungen Uber Allgemeneine Pathogie.Vol 1. Berlin: Hirschwald, 1877: 136.

5 Effeney DJ, Krupski WC. Paradoxical embolism in pregnancy: an usual thromboembolic event. West $f \mathrm{Med}$ 1984;140:287-8

6 Caplan LR, Hier DB, D'Cruz I. Cerebral embolism in the Michael Reese Stroke Registry. Stroke 1983;14:530-6.

7 Lechat P, Mas JI, Lascault G, et al. Prevalence of patent

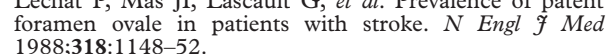

8 Hagen PT, Scholz DG, Edwards WD. Incidence and size of patent foramen ovale during the first 10 decades of life: an autopsy study of 965 normal hearts. Mayo Clin Proc autopsy study

9 Dizon-Townson D, Magee KP, Twickler DM, et al. Coarctation of the abdominal aorta in pregnancy: diagnosis by magnetic resonance imaging. Obstet Gynecol 1995;85(5 pt 2):817-19

10 Turrentine MA, Braems G, Ramirez MM. Use of thrombolytics for the treatment of thromboembolic disease during pregnancy. Obstet Gynecol Surv 1995;50:534-41. 


\section{Severe symptomatic hypercalcaemia}

Q1: What is your differential diagnosis for this case?

The highly sensitive and specific immunometric assays for intact parathyroid hormone (PTH) separates hyperparathyroidism from all other causes of hypercalcaemia. ${ }^{1}$ With few exceptions, non-parathyroid causes of hypercalcaemia are accompanied by low serum concentrations of intact PTH. Chronic treatment with lithium may produce hypercalcaemia that is associated with high serum intact $\mathrm{PTH}$, a clinical picture indistinguishable from primary hyperparathyroidism. ${ }^{2}$ Familial hypocalciuric hypercalcaemia must be considered in healthy patients who have had hypercalcaemia since the first decade of life; they usually have hypocalciuria and normal serum concentrations of intact PTH. ${ }^{3}$

A solitary parathyroid adenoma is the underlying pathology in more than $80 \%$ of cases of primary hyperparathyroidism. ${ }^{45}$ Diffuse hyperplasia of all parathyroid glands occurs in about $15 \%-20 \%$ of patients and may in about half of these be part of a multiple endocrine neoplasia (MEN). ${ }^{1}$ Multiple adenomas and parathyroid cysts are uncommon, and parathyroid carcinoma is very rare $(<1 \%) .{ }^{1}$ Acute primary hyperparathyroidism is an unusual form of the disease characterised by life threatening hypercalcaemia. ${ }^{67}$

\section{Q2: What further investigations would you perform?}

Ultrasonography demonstrated a nodule of 3 $\mathrm{cm}$ diameter in the left lower parathyroid gland. Abdominal radiography and ultrasonography did not reveal nephrocalcinosis or nephrolithiasis. Bone series were normal. Plasma calcitonin was $29 \mathrm{ng} / \mathrm{l}$ (normal $<50 \mathrm{ng} / \mathrm{l}$ ) and fasting plasma gastrin was $44 \mathrm{ng} / 1$ (normal $<150 \mathrm{ng} / \mathrm{l}$ ). The 24 hour urinary free catecholamines, dopamine, epinephrine, and norepinephrine were normal. In our case, there were no family history, clinical or laboratory findings of MEN 1 (primary hyperparathyroidism, tumours of the pituitary and pancreas, often associated with Zollinger-Ellison syndrome) or MEN 2A (primary hyperparathyroidism, pheochromocytoma, and medullary carcinoma of the thyroid).

\section{Q3: What is the therapy for the severe hypercalcaemia in this case?}

The patient was treated with saline rehydration, low doses of intravenous frusemide (after rehydration), intravenous clodronate, and subcutaneous calcitonin. Four days after, the blood calcium was $2.2 \mathrm{mmol} / 1$ and the creatinine was $61.9 \mu \mathrm{mol} / 1$. Parathyroid surgical exploration revealed an enlarged left lower parathyroid gland. The other three glands were normal. Removal of the left lower parathyroid gland was performed, and the histopathological examination demonstrated a $3 \mathrm{~cm}$ diameter parathyroid adenoma. Seven days after surgery, intact PTH was $43 \mu \mathrm{g} / 1$ and blood calcium remained normal. Six months later, the patient remains asymptomatic and blood calcium and intact PTH are normal.

Surgery, with its risks, for all patients with primary hyperparathyroidism now seems unwise when many will have no features of metabolic bone or renal disease. ${ }^{8}$ When done by an experienced parathyroid surgeon, parathyroidectomy is curative in more than $90 \%$ of cases. ${ }^{1}$ Medical treatment is intended to lower blood calcium to less dangerous levels. However, it is not necessary to obtain normal levels of calcium, and surgery must be carried out as soon as the patients's clinical condition and metabolism improve sufficiently. ${ }^{7}$ Emergency neck exploration should be reserved for unusual patients in whom hypercalcaemia cannot be controlled medically and the clinical picture is severe.

\section{Discussion}

Hypercalcaemia in an adult who is asymptomatic is usually due to primary hyperparathyroidism and severe hypercalcaemia suggests cancer or parathyroid carcinoma. ${ }^{1}$ The clinical picture of our case suggests malignancy. Muscle weakness is not common in primary hyperparathyroidism but is common in acute primary hyperparathyroidism, due to the severe hypercalcaemia. ${ }^{67}$ The serum creatinine wasn't particularly raised, which is a surprise in view of the very high level of serum calcium; this suggests that our patient's hypercalcaemia was of short duration. The proportion of symptom-free patients with primary hyperparathyroidism has increased since the introduction of the multichannel autoanalyser. ${ }^{45}$ Our case had acute primary hyperparathyroidism caused by a parathyroid adenoma, an unusual form of the disease. Acute primary hyperparathyroidism, also called parathyroid intoxication, parathyroid storm or parathyroid crisis, is characterised by symptomatic marked hypercalcaemia with very high serum PTH levels and with polyuria, dehydration, reduced renal function, and worsening hypercalcaemia. ${ }^{67}$ Most cases of acute primary hyperparathyroidism are due to a parathyroid adenoma. ${ }^{67}$ Some authors do not exclude the parathyroid carcinoma from the acute primary hyperparathyroidism. ${ }^{7}$ Remarkable increases of PTH are characterisitc of acute primary hyperparathyroidism, up to values 30 times normal levels. ${ }^{6}$ It has excluded autonomous PTH secretion as a possible cause of acute primary hyperparathyroidism, and it has been suggested that a sudden increase in the set point of the diseased parathyroid cells in the presence of a huge cell mass accounts in large part for both the marked hypercalcaemia and elevated PTH levels in these patients. ${ }^{9}$ Infections, recent surgery, immobilisation, dehydration, and trauma appear to play a prominent part in the acute primary hyperparathyroidism. ${ }^{67}$

Frusemide must be used for therapy of the hypercalcaemia after rehydration and with caution as it counteracts the effects of rehydration. Saline rehydration reverses the increased proximal tubular calcium reabsorption, and calcitonin inhibits the distal tubular calcium reabsorption. 


\section{Learning points}

- Hypercalcaemia with normal or high PTH levels occurs in primary hyperparthyroidism, familial hypocalciuric hypercalcaemia and chronic treatment with lithium

- Acute primary hyperparthyroidism is an unusual form of the disease characterised by life threatening hypercalcaemia

- Severe hypercalcaemia suggests cancer or parathyroid carcinoma, and acute primary hyperparthyroidism constitutes an exception of this assertion

Final diagnosis

Acute primary hyperparathyroidism, caused by a solitary parathyroid adenoma.
1 Potts JT Jr. Diseases of the parathyroid gland and other hyper- and hypocalcemic disorders. In: Fauci AS, Braunwald E, eds. Harrison's principles of internal medicine. New McGraw-Hill, 1998: 2227-47.

2 Mallete LE, Khouri K, Zengotita $\mathrm{H}$, et al. Lithium treatment increases intact and midregion parathyroid hormone and parathyroid volume. 7 Clin Endocrinol Metab 1989;68:65460.

3 Heath HI. Familial benign (hypocalciuric) hypercalcemia: a troublesome mimic of mild primary hyperparathyroidism. Endocrinol Metab Clin North Am 1989;18:723-40.

4 Heath HWI, Hodgson SF, Kennedy MA. Primary hyperparathyroidism: incidence, morbidity and potential hyperparathyroidism: incidence, morbidity and potential economic

5 Mollerup CL, Bollerslev J, Blichert-Toft M. Primary hyperparathyroidism: incidence and clinical and biochemical characteristics: a demographic study. Eur $\mathcal{f}$ Surg 1994;160:485-9.

6 Fitzpatrick LA, Bilezikian JP. Acute primary hyperparathyroidism. Am f Med 1987;82:275-82.

7 Sarfati E, Desportes L, Gossot D, et al. Acute primary hyperparathyroidism: experience of 59 cases. $\mathrm{Br}$ f Surg 1989;76:979-81.

8 Potts JT Jr, Fradkin JE, Aurbach GD, et al. Proceedings of the NIH Consensus Development Conference on Diagnosis and Management of Asymptomatic Primary Hyperparathyand Management of Asymptomatic Primary Hyperpa

9 Schachter PP, Christy MD, Avigad IS, et al. Non-autonomy of parathyroid hormone secretion in acute primary hyperparathyroidism. Clin Endocrinol (Oxf) 1992;37:565-9. 